\title{
Decapado del acero inoxidable AISI 316L utilizando una mezcla ecológica
} de $\mathrm{H}_{2} \mathrm{O}_{2}-\mathrm{H}_{2} \mathrm{SO}_{4}-\mathrm{HF}$

\author{
L. Narváez ${ }^{*}$ E. Cano**, D.M. Bastidas** y P.P. Gómez**
}

\begin{abstract}
Resumen En la presente investigación se estudia el decapado del acero inoxidable AISI 316L utilizando una mezcla de agua oxigenada $\left(\mathrm{H}_{2} \mathrm{O}_{2}\right)$ y los ácidos sulfúrico $\left(\mathrm{H}_{2} \mathrm{SO}_{4}\right)$ y fluorhídrico (HF) a pH 2,0. La estabilidad de la mezcla $\mathrm{H}_{2} \mathrm{O}_{2}-\mathrm{H}_{2} \mathrm{SO}_{4}$ - $\mathrm{HF}$ se ha ensayado variando el contenido de iones férrico de 0 a $40 \mathrm{~g} / \mathrm{l}$ y la temperatura de 25 a $60{ }^{\circ} \mathrm{C}$. La velocidad de decapado a $50{ }^{\circ} \mathrm{C}$ ha sido de 2,6 y $0,2 \mathrm{mg} / \mathrm{dm}^{2}$ día (mdd), en ausencia y presencia de $40 \mathrm{~g} / \mathrm{l}$ de iones férrico, respectivamente. Se ha utilizado el ácido p-toluen sulfónico como estabilizante del $\mathrm{H}_{2} \mathrm{O}_{2}$.
\end{abstract}

Palabras clave Decapado acero inoxidable AISI 316L. Agua oxigenada. p-Toluen sulfónico.

\section{Pickling of AISI 316 stainless steel using and ecological mixture of $\mathrm{H}_{2} \mathrm{O}_{2}-\mathrm{H}_{2} \mathrm{SO}_{4}-\mathrm{HF}$}

\begin{abstract}
This study reports the pickling of austenitic AISI 316L stainless steel (SS) using a mixture of hydrogen peroxide $\left(\mathrm{H}_{2} \mathrm{O}_{2}\right)$, sulphuric acid $\left(\mathrm{H}_{2} \mathrm{SO}_{4}\right)$ and hydrofluoric acid $(\mathrm{HF})$ at $\mathrm{pH}$ 2.0. The stability of $\mathrm{H}_{2} \mathrm{O}_{2}$ was also studied using different concentrations of ferric ion from 0 to $40 \mathrm{~g} / \mathrm{l}$ and temperature from 25 to $60^{\circ} \mathrm{C}$. The pickling rate at $50^{\circ} \mathrm{C}$ in the presence and absence of $40 \mathrm{~g} / \mathrm{l}$ ferric ion was 2.6 and $0.2 \mathrm{mg} / \mathrm{dm}^{2}$ day (mdd), respectively. $\mathrm{p}$-Toluene sulphonic acid was used as stabilizer of $\mathrm{H}_{2} \mathrm{O}_{2}$.
\end{abstract}

Keywords Pickling AISI 316L stainless steel. Hydrogen peroxide. p-Toluene sulphonic acid.

\section{INTRODUCCIÓN}

En general, el tratamiento de decapado del acero inoxidable comprende varias etapas: el recocido en una atmósfera adecuada; la eliminación de las capas de óxidos, una interna de óxido ferroso $(\mathrm{FeO})$, una intermedia de óxido magnético $\left(\mathrm{Fe}_{3} \mathrm{O}_{4}\right)$ y una externa de óxido férrico $\left(\mathrm{Fe}_{2} \mathrm{O}_{3}\right)$; la disolución de la capa empobrecida en cromo; y la recuperación de las propiedades pasivas de la capa superficial del acero inoxidable ${ }^{[1]}$.

Los baños convencionales de decapado del acero inoxidable utilizan mezclas de los ácidos nítrico $\left(\mathrm{HNO}_{3}\right)$ y fluorhídrico (HF), las cuales presentan el inconveniente de que generan gran cantidad de gases, designados de forma genérica como Nox: óxido nítrico $(\mathrm{NO})$ y dióxido de nitrógeno $\left(\mathrm{NO}_{2}\right)$, principalmente; y, en menor cantidad, tetróxido de nitrógeno $\left(\mathrm{N}_{2} \mathrm{O}_{4}\right)$ y trióxido de nitrógeno $\left(\mathrm{N}_{2} \mathrm{O}_{3}\right)$. Dichos gases son tóxicos ${ }^{[2]}$.

El objetivo de esta comunicación es estudiar el decapado del acero inoxidable AISI 316L utilizando una mezcla ecológica de $\mathrm{H}_{2} \mathrm{O}_{2}$ y los ácidos $\mathrm{H}_{2} \mathrm{SO}_{4}$ y HF. Se estudia la influencia de la temperatura y el contenido de iones férrico en la estabilidad del agua oxigenada.

\section{MATERIALES Y MÉTODOS}

El material ensayado ha sido acero inoxidable austenítico AISI 316L, de composición (\% en peso): $<0,0039 \mathrm{C} ; \leq 0,75 \mathrm{Si} ; \leq 2,20 \mathrm{Mn} ; 0,040 \mathrm{P} ; 0,015$ $\mathrm{S} ; 17,8 \mathrm{Cr} ; 12,5 \mathrm{Ni} ; 2,8 \mathrm{Mo}$; y el resto hierro $(\mathrm{Fe})$. Se partió de material en forma de chapa laminada en frío de $2 \mathrm{~mm}$ de espesor. La chapa se cortó en probetas de dimensiones $2,5 \mathrm{~cm} \times 7,5 \mathrm{~cm}(37,9$

${ }^{*}$ Instituto de Metalurgia, UASLP, Avda. Sierra Leona 550, 78210 San Luis Potosí, México.

$\left(^{* *}\right) \quad$ Centro Nacional de Investigaciones Metalúrgicas, CSIC, Avda. Gregorio del Amo 8, 28040 Madrid.

$\left(^{* * *}\right)$ School of Chemistry, University of St. Andrews, St. Andrews, Fife KY16 9ST, Scotland, United Kingdom. 
$\mathrm{cm}^{2}$ de superficie). Las probetas se desengrasaron con acetona en un baño ultrasónico, se lavaron con agua destilada y se secaron con aire a presión.

Se utilizó como agente estabilizante del agua oxigenada el ácido p-toluen sulfónico monohidratado (APTS) $\left(\mathrm{C}_{6} \mathrm{H}_{4} \mathrm{HSO}_{3} \mathrm{CH}_{3} \cdot \mathrm{H}_{2} \mathrm{O}\right)$. El APTS se disolvió previamente en alcohol etílico $\left(\mathrm{C}_{2} \mathrm{H}_{5} \mathrm{OH}\right)$ a temperatura ambiente. La relación en peso de APTS: $\mathrm{H}_{2} \mathrm{O}_{2}$ fue de 5:100 [3].

La composición de la mezcla decapante fue: 30 g/l de $\mathrm{H}_{2} \mathrm{O}_{2}$ estabilizada, $75 \mathrm{~g} / \mathrm{l}$ de $\mathrm{H}_{2} \mathrm{SO}_{4}, 25 \mathrm{~g} / \mathrm{l}$ de HF y $20 \mathrm{~g} / \mathrm{l}$ de sulfato férrico $\left(\mathrm{Fe}_{2}\left(\mathrm{SO}_{4}\right)_{3}\right)$. A un volumen determinado de agua destilada se adicionaron los compuestos $\mathrm{H}_{2} \mathrm{SO}_{4}$, $\mathrm{HF}$ y $\mathrm{Fe}_{2}\left(\mathrm{SO}_{4}\right)_{3}$ hasta su completa disolución, dejando enfriar la mezcla a temperatura ambiente. Por otro lado, se preparó el agua oxigenada estabilizada. Finalmente, se introdujeron los compuestos en un matraz hasta un volumen de $1.000 \mathrm{ml}$.

Los ensayos se realizaron a $\mathrm{pH} \mathrm{2,0} \mathrm{conseguido}$ mediante la adición de $\mathrm{H}_{2} \mathrm{SO}_{4}$. La mezcla decapante se ensayó a las temperaturas de $25,40,50$ y $60^{\circ} \mathrm{C}$.

La pérdida de $\mathrm{H}_{2} \mathrm{O}_{2}$ (\% peso) se realizó mediante valoración con permanganato potásico $\left(\mathrm{KMnO}_{4}\right)$ de concentración $0,1 \mathrm{~N}$. Se extrajeron 5 $\mathrm{ml}$ de la mezcla decapante, se adicionaron $100 \mathrm{ml}$ de agua destilada y $5 \mathrm{ml}$ de $\mathrm{H}_{2} \mathrm{SO}_{4}$ y se valoraron con $\mathrm{KMnO}_{4}$ hasta obtener una coloración rosa permanente ${ }^{[4-6]}$.

La velocidad de decapado se llevó a cabo utilizando la técnica gravimétrica, se introdujeron las probetas de AISI 316L en un volumen de $250 \mathrm{ml}$ de la mezcla decapante. La pérdida de peso se determinó por diferencia de pesada al inicio y al final del ensayo, $1 \mathrm{~h}$. Se utilizó una balanza analítica electrónica con una precisión de $\pm 0,1 \times 10^{-3} \mathrm{~g}$. Los ensayos de microscopía electrónica (MEB) se realizaron con el equipo JEOL JXA-840. Se trabajó a un voltaje de $20 \mathrm{kV}$ y se realizaron micrografías con el detector de electrones secundarios. La distancia de trabajo fue de $10 \mathrm{~mm}$.

\section{RESULTADOS Y DISCUSIÓN}

La figura 1 muestra la estabilidad del $\mathrm{H}_{2} \mathrm{O}_{2}$ frente al tiempo. Se observa que, en el intervalo de temperatura de $40-60{ }^{\circ} \mathrm{C}$ la pérdida de $\mathrm{H}_{2} \mathrm{O}_{2}$ es en torno al $80 \%$ durante un tiempo de $60 \mathrm{~min}$. Por el contrario, a $25^{\circ} \mathrm{C}$, para que la pérdida de $\mathrm{H}_{2} \mathrm{O}_{2}$ sea del $80 \%$ se requiere un tiempo de $13 \mathrm{~h}$.

Es conocido que la presencia de iones férrico favorece la descomposición del peróxido de hidróge-

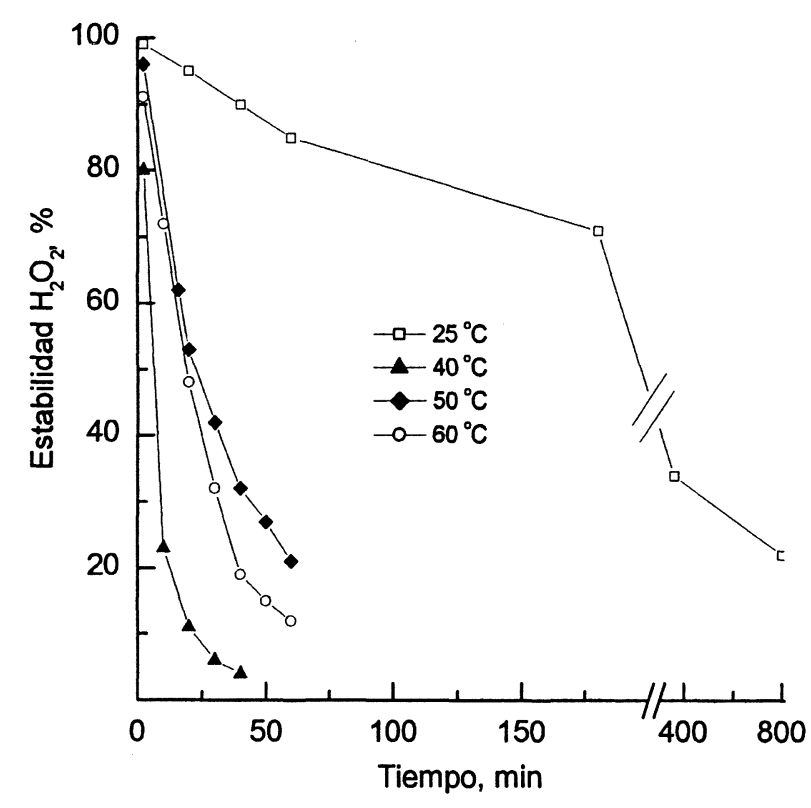

Figura 1. Estabilidad del $\mathrm{H}_{2} \mathrm{O}_{2}$ frente al tiempo en función de la temperatura.

Figure 1. $\mathrm{H}_{2} \mathrm{O}_{2}$ stability vs. time in function of temperature.

no. Así, se realizaron ensayos sumergiendo una probeta de AISI 316L (37,9 $\mathrm{cm}^{2}$ de superficie) en un matraz con $250 \mathrm{ml}$ de $\mathrm{H}_{2} \mathrm{O}_{2}$ de concentración $20 \mathrm{~g} / \mathrm{l}$ y a $25^{\circ} \mathrm{C}$. La figura 2 muestra los resultados obtenidos de estabilidad del $\mathrm{H}_{2} \mathrm{O}_{2}$ frente al tiempo.

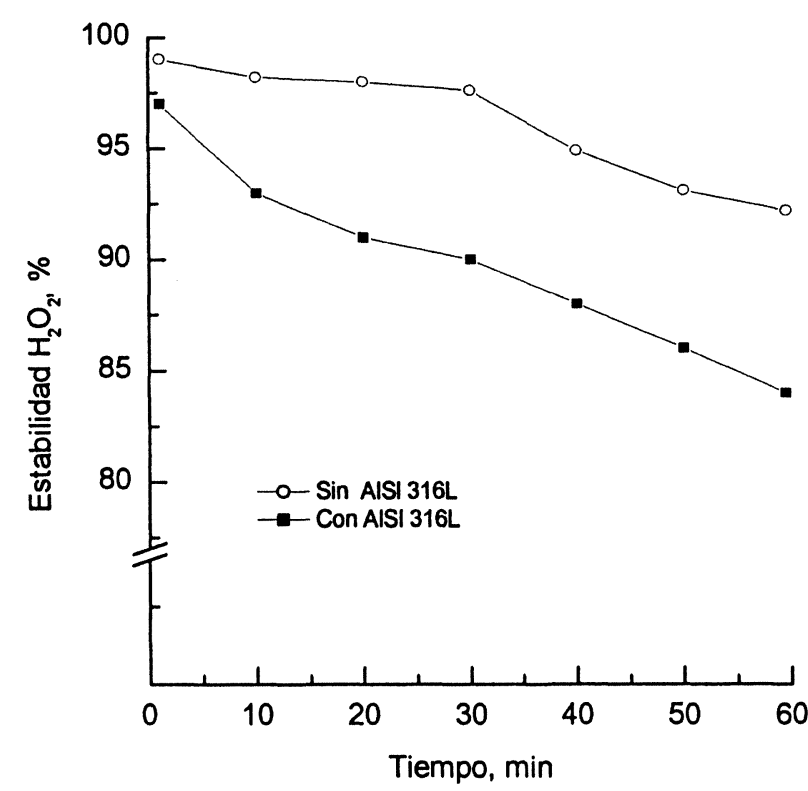

Figura 2. Estabilidad del $\mathrm{H}_{2} \mathrm{O}_{2}$ frente al tiempo con y $\sin$ AISI 316L.

Figure 2. $\mathrm{H}_{2} \mathrm{O}_{2}$ stability in the presence and absence of AISI $316 L$ SS.

Rev. Metal. Madrid Vol. Extr. (2005) 417-422 
Se observa que la pérdida de $\mathrm{H}_{2} \mathrm{O}_{2}$ en ausencia de AISI 316L es del orden del $7 \%$ para un tiempo de $60 \mathrm{~min}$. Por el contrario, al introducir el acero inoxidable AISI 316L la pérdida de $\mathrm{H}_{2} \mathrm{O}_{2}$ es $~ 17 \%$.

La figura 3 muestra la influencia de la presencia del compuesto APTS en la estabilidad del $\mathrm{H}_{2} \mathrm{O}_{2}$ frente al tiempo de ensayo. En ausencia de APTS la pérdida de $\mathrm{H}_{2} \mathrm{O}_{2}$ es muy importante, después de $4 \mathrm{~d}$ de ensayo se ha perdido $\sim 90 \%$. Por el contrario, en presencia de APTS y para los primeros $4 \mathrm{~d}$ de ensayo, la estabilidad del $\mathrm{H}_{2} \mathrm{O}_{2}$ es en torno al $80 \%$.

En la bibliografía se recomienda que el estabilizante del peróxido de hidrógeno esté constituido por un ácido y una solución alcohólica ${ }^{[3-5]}$.

En la presente investigación se realizaron ensayos utilizando como disolvente el alcohol etílico. Se prepararon dos baños, uno, adicionando el estabilizante APTS sólido 1,5\% (relación en peso) y otro, añadiendo el APTS previamente disuelto en etanol. La temperatura se mantuvo a $50{ }^{\circ} \mathrm{C}$ y se utilizó $\mathrm{H}_{2} \mathrm{O}_{2}$ de concentración $30 \mathrm{~g} / \mathrm{l}$. La figura 4a) muestra el aspecto superficial de la probeta decapada, adicionando el APTS sólido. Se observa que algunos granos presentan un ligero ataque y en forma localizada. La figura 4b) muestra el aspecto superficial de una probeta en la que se disolvió el APTS en etanol. Se observa una mejor apariencia superficial y un ataque más homogéneo que en la Fig. 4a).

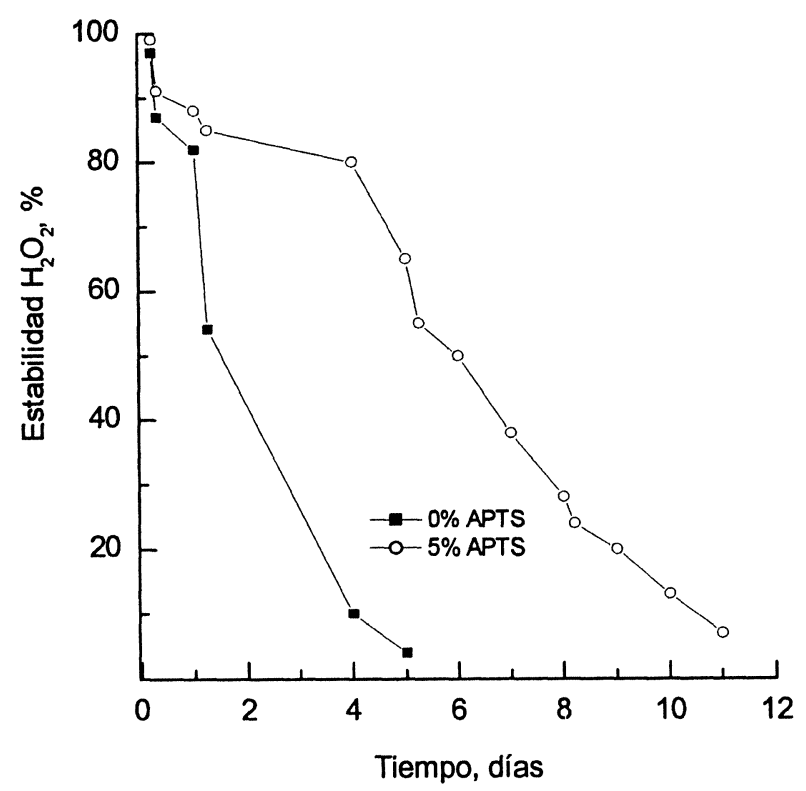

Figura 3. Estabilidad del $\mathrm{H}_{2} \mathrm{O}_{2}$ frente al tiempo con y $\sin 5$ $\%$ de APTS.

Figure 3. $\mathrm{H}_{2} \mathrm{O}_{2}$ stability in the presence and absence of $5 \%$ APTS.

Rev. Metal. Madrid Vol. Extr. (2005) 417-422
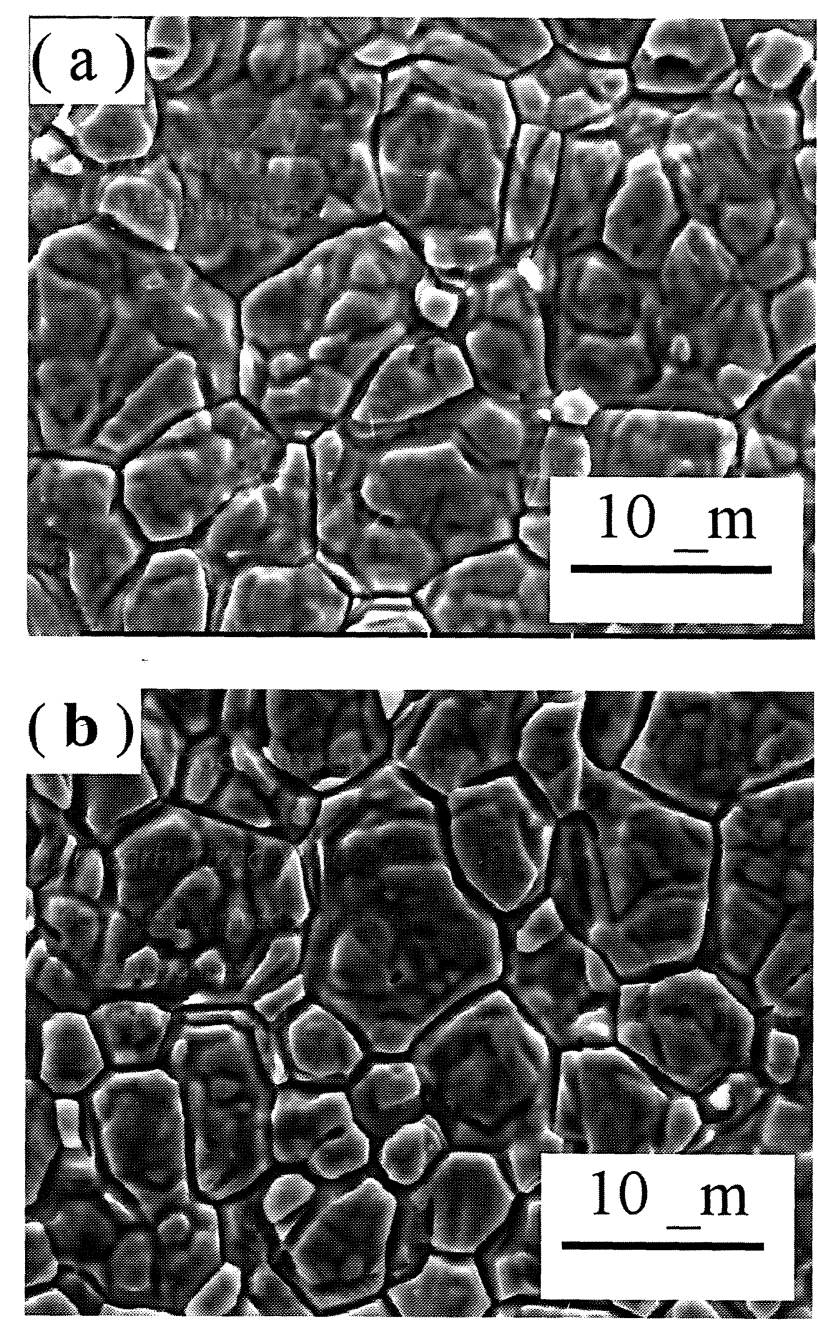

Figura 4. Aspecto superficial del AISI 316L: (a) APTS sólido y (b) APTS disuelto en etanol.

Figure 4. Superficial aspect of AISI 316 L SS: (a) solid APTS and (b) APTS dissolved in ethanol.

La figura 5 muestra la evolución de la mezcla ecológica $\left(\mathrm{H}_{2} \mathrm{O}_{2}-\mathrm{H}_{2} \mathrm{SO}_{4}\right.$ - $\left.\mathrm{HF}\right)$ con el tiempo, utilizando distintas concentraciones de APTS y en presencia de una probeta de AISI $316 \mathrm{~L}\left(37,9 \mathrm{~cm}^{2}\right.$ de superficie) y a $50^{\circ} \mathrm{C}$. Se observa que, utilizando 1,5\% de APTS, la descomposición de la mezcla decapante tiene lugar en un tiempo de $45 \mathrm{~min}$. A mayores concentraciones de APTS, 3-10\%, se obtiene un mejor comportamiento de la mezcla decapante, siendo las pérdidas de agua oxigenada del 15 al $25 \%$.

La figura 6 muestra la estabilidad del $\mathrm{H}_{2} \mathrm{O}_{2}$ con el tiempo en presencia de AISI 316L $\left(37,9 \mathrm{~cm}^{2}\right)$ con un concentración de $\mathrm{H}_{2} \mathrm{O}_{2}$ de $20 \mathrm{~g} / \mathrm{l}$, en ausencia y presencia de $5 \%$ APTS y a $50{ }^{\circ} \mathrm{C}$ (Fig. 6a) y a 20,50 y $60^{\circ} \mathrm{C}$ (Fig. 6b), mientras que para 30 y $40 \mathrm{~g} / \mathrm{l}$ de iones férrico se produce una perdida de $\mathrm{H}_{2} \mathrm{O}_{2}$ de $\sim 90 \%$ en 20 min. 


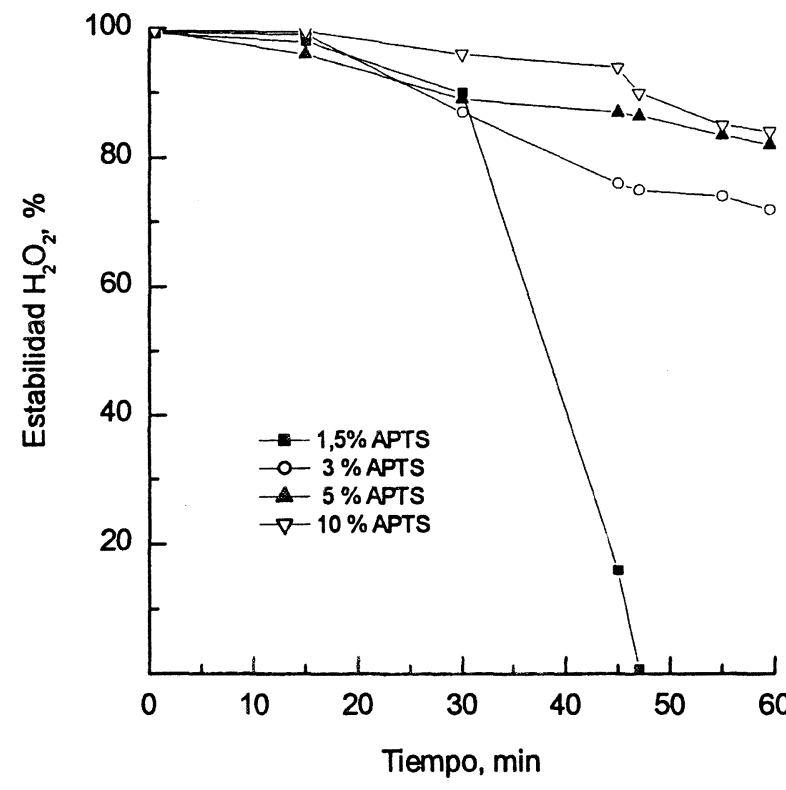

Figura 5. Estabilidad del $\mathrm{H}_{2} \mathrm{O}_{2}$ frente al tiempo en función del contenido de APTS.

Figure 5. $\mathrm{H}_{2} \mathrm{O}_{2}$ stability vs. time in function of APTS.

Se observa que, para un tiempo de $60 \mathrm{~min}$, la solución decapante en ausencia de PTSA presenta una pérdida de la estabilidad del $90 \%$, mientras que, en presencia de PTSA, la pérdida es sólo del $17 \%$. A medida que aumenta la temperatura, la estabilidad del $\mathrm{H}_{2} \mathrm{O}_{2}$ también aumenta (Fig. 6b).

El $\mathrm{H}_{2} \mathrm{O}_{2}$ tiene la función de favorecer la reacción de oxidación del ion ferroso a ion férrico. $\mathrm{Pa}$ ra conocer la influencia del $\mathrm{H}_{2} \mathrm{O}_{2}$ en la mezcla decapante, se realizaron ensayos utilizando $20,30 \mathrm{y}$ $40 \mathrm{~g} / \mathrm{l}$ de $\mathrm{H}_{2} \mathrm{O}_{2}$. La figura 7 muestra que, para un tiempo de $60 \mathrm{~min}$, con la menor concentración de $\mathrm{H}_{2} \mathrm{O}_{2}$ ensayada se produce la mayor pérdida de estabilidad del $\mathrm{H}_{2} \mathrm{O}_{2}$.

La figura 8 muestra la variación de la estabilidad del $\mathrm{H}_{2} \mathrm{O}_{2}$ en función del tiempo, en presencia de distintas concentraciones de iones férrico $\left[\mathrm{Fe}_{2}\left(\mathrm{SO}_{4}\right)_{3}\right]$ y a $50{ }^{\circ} \mathrm{C}$. Se observa que la pérdida de $\mathrm{H}_{2} \mathrm{O}_{2}(30 \mathrm{~g} / \mathrm{l})$ aumenta en la medida que se incrementa el contenido de iones férrico; a concentraciones menores o iguales a $20 \mathrm{~g} / \mathrm{l}$ de iones férrico la pérdida de $\mathrm{H}_{2} \mathrm{O}_{2}$ es pequeña, requiriendo 60 min para una perdida de $\sim 30 \%$.

Estos resultados permiten definir un umbral de concentración de iones férrico $(20 \mathrm{~g} / \mathrm{l})$, por encima del cual la pérdida de $\mathrm{H}_{2} \mathrm{O}_{2}$ aumenta drásticamente. Esta pérdida de $\mathrm{H}_{2} \mathrm{O}_{2}$ se puede atribuir a su descomposición catalítica por la presencia de iones férrico $^{[2 \text { y } 7-10]}$. En la mezcla decapante tienen lugar varias reacciones ${ }^{[11]}$ :

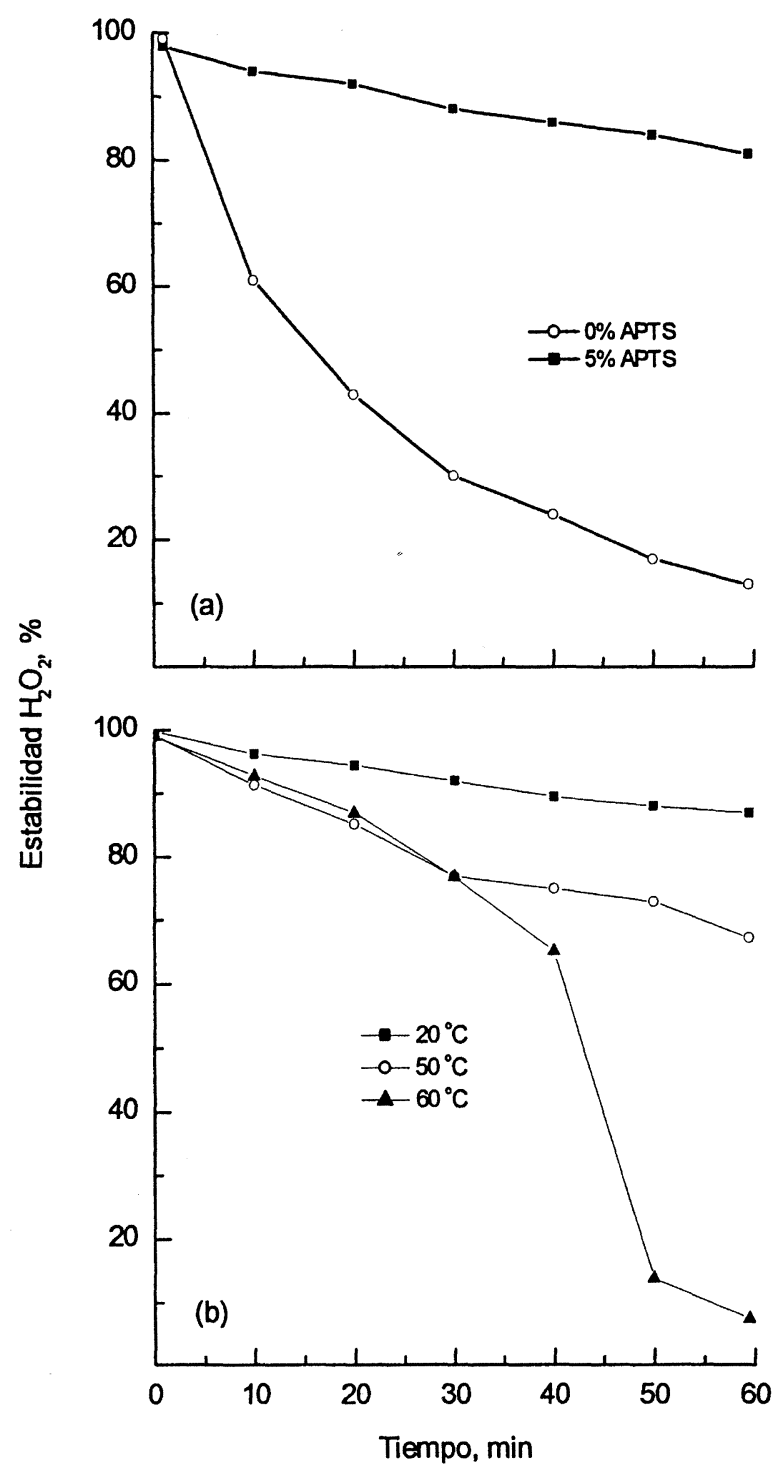

Figura 6. Estabilidad del $\mathrm{H}_{2} \mathrm{O}_{2}$ frente al tiempo con y $\sin$ APTS y en función de la temperatura.

Figure 6. $\mathrm{H}_{2} \mathrm{O}_{2}$ stability in presence and absence of APTS and in function of temperature.

$\mathrm{FeO}+\mathrm{H}_{2} \mathrm{SO}_{4} \rightarrow \mathrm{FeSO}_{4}+\mathrm{H}_{2} \mathrm{O}$

$\mathrm{Fe}_{2} \mathrm{O}_{3}+3 \mathrm{H}_{2} \mathrm{SO}_{4} \rightarrow \mathrm{Fe}_{2}\left(\mathrm{SO}_{4}\right)_{3}+3 \mathrm{H}_{2} \mathrm{O}$

$\mathrm{Fe}_{3} \mathrm{O}_{4}+4 \mathrm{H}_{2} \mathrm{SO}_{4} \rightarrow \mathrm{FeSO}_{4}+\mathrm{Fe}_{2}\left(\mathrm{SO}_{4}\right)_{3}+4 \mathrm{H}_{2} \mathrm{O}$

$\mathrm{Fe}+\mathrm{H}_{2} \mathrm{SO}_{4} \rightarrow \mathrm{FeSO}_{4}+\mathrm{H}_{2}$

La figura 9 muestra la velocidad de decapado, $\mathrm{mg} / \mathrm{dm}^{2}$ día (mdd), del acero inoxidable AISI 316L utilizando una mezcla de composición: $30 \mathrm{~g} / \mathrm{l}$ $\mathrm{H}_{2} \mathrm{O}_{2}, 75 \mathrm{~g} / \mathrm{l} \mathrm{H}_{2} \mathrm{SO}_{4}, 25 \mathrm{~g} / \mathrm{l} \mathrm{HF}, 5$ \% PTSA, $\mathrm{pH}$ 2,0 , en función de la concentración de iones férrico, añadido en forma de $\mathrm{Fe}_{2}\left(\mathrm{SO}_{4}\right)_{3}$ y a $50^{\circ} \mathrm{C}$. El tiempo de ensayo fue de $1 \mathrm{~h}$. Se puede observar que la velocidad de decapado del AISI 316L disminuye a medida que aumenta el contenido de iones férrico en la solución. 


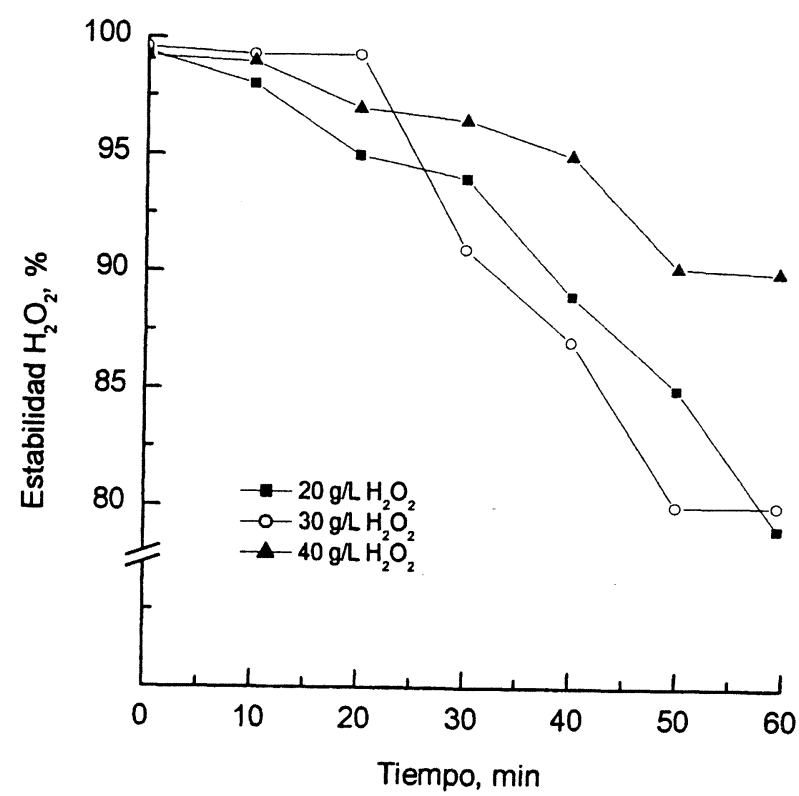

Figura 7. Estabilidad del $\mathrm{H}_{2} \mathrm{O}_{2}$ frente al tiempo en presencia de AISI 316L.

Figure 7. $\mathrm{H}_{2} \mathrm{O}_{2}$ stability vs. time in the presence of AISI $316 \mathrm{~L}$ SS.

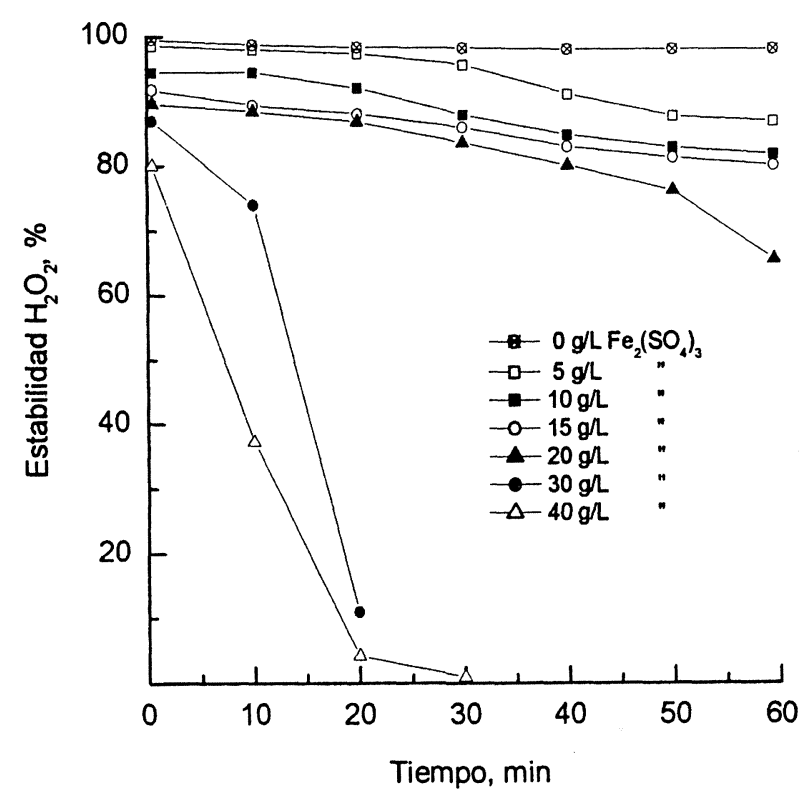

Figura 8. Estabilidad del $\mathrm{H}_{2} \mathrm{O}_{2}$ frente al tiempo en función del contenido de ión férrico.

Figure 8. $\mathrm{H}_{2} \mathrm{O}_{2}$ stability vs. time in function of ferric ion.

\section{CONCLUSIONES}

Los iones férrico y la temperatura tienen una influencia negativa en la estabilidad del agua oxigenada. Se puede definir un umbral de concentración de iones férrico, en torno a $20 \mathrm{~g} / \mathrm{l}$, por encima

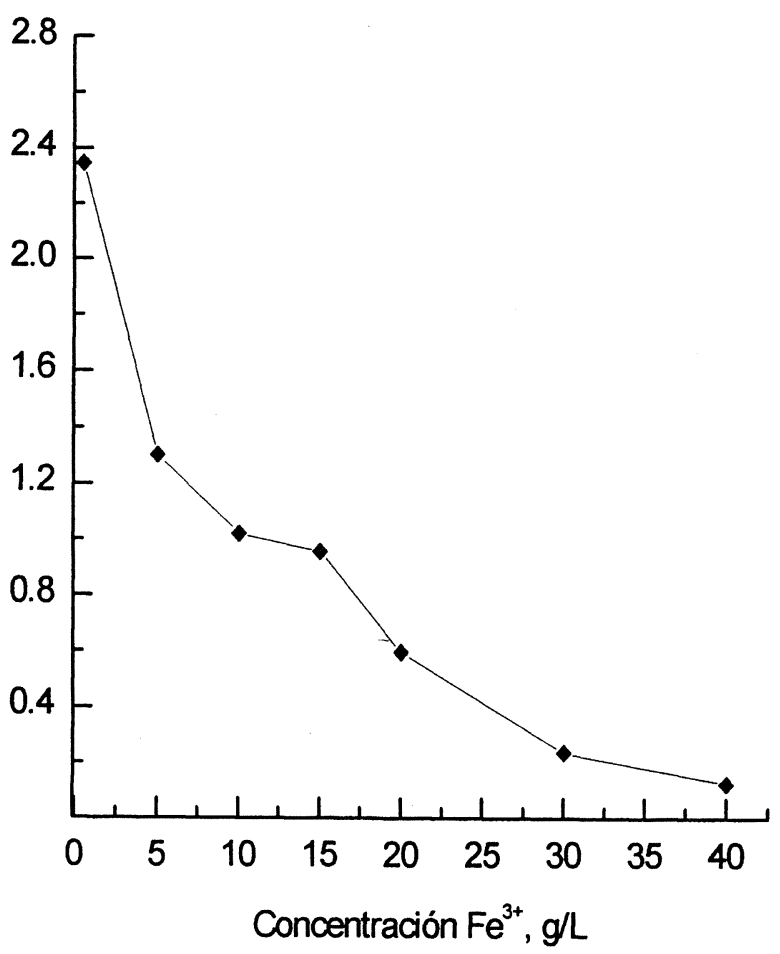

Figura 9. Velocidad de decapado del acero inoxidable AISI $316 \mathrm{~L}$ frente a la concentración de ion férrico.

Figure 9. Pickling rate of AISI 316L SS vs. ferric ion.

del cual la pérdida de agua oxigenada aumenta considerablemente. Se ha observado que, en el intervalo de $40-60^{\circ} \mathrm{C}$ de temperatura, la pérdida de agua oxigenada es en torno al $80 \%$, para un tiempo de ensayo de $60 \mathrm{~min}$.

El ácido p-toluen sulfónico (APTS) es un buen estabilizante del agua oxigenada, produciéndose una pérdida de esta última del $60 \%$ después de $12 \mathrm{~d}$ de ensayo.

La velocidad de decapado del acero inoxidable AISI 316L depende de la concentración de iones férrico presentes en la mezcla decapante.

\section{Agradecimientos}

Los autores desean expresar su agradecimiento a la CICYT por la financiación del Proyecto No. MAT2001-1732-C02- 01 y a la Empresa Acerinox SA por el suministro del acero inoxidable AISI 316L ensayado.

\section{REFERENCIAS}

[1] L. Narváez, E. Cano y J.M. Bastidas, Mater. Corros. 54 (2003) 84-87.

[2] N.J. SAnders, Anti-Corros. Methods Mater. 44 (1997) 20 25. 
Decapado del acero inoxidable AISI $316 \mathrm{~L}$ utilizando una mezcla ecológica de $\mathrm{H}_{2} \mathrm{O}_{2}-\mathrm{H}_{2} \mathrm{SO}_{4}$ - $\mathrm{HF}$

L. NARVÁEZ, E. CANO, D.M. BASTIDAS Y P.P. GÓMEZ

[3] S.J. Colgan, N.J. Sanders y C.F. Mcdonogh, Patente USA, US6126755, 2000.

[4] P.L. Moratill.A, Patente Española EP2099027, 1997.

[5] M. BIANCHI, Patente Europea EO0505606, 1992.

[6] S. Masaki, K. Yoshikasu, I. Katsutoshi, K. Toshilchi y F. TADAHIRO, Patente Japonesa JP11061465, 1999.

[7] L.E. MoneEs, Met. Prog. 119 (1981) 36-38.
[8] M. ZaVATtoni, Patente Europea, 0795628 B1, 1997.

[9] L.A. Godínez, Y. Meas, R. Ortiga-Borges y A. Corona, Rev. Metal. Madrid 30 (2003) 140-158.

[10] O.V. Correa, M. Saiki, S.O. Rogero E I. Costa, Rev. Metal. Madrid Vol. Extr. (2003) 91-96.

[11] L. Rozenfeld, Corrosion Inhibitors, McGraw-Hill Internacional, Nueva York, pp. 207-211, 1981. 